\title{
Modeling Enhanced Performances by Optical Nanostructures in Water-Splitting Photoelectrodes
}

\author{
Luc Driencourt, Benjamin Gallinet,* Catherine E. Housecroft, Sören Fricke, and Edwin C. Constable*
}

Cite This: J. Phys. Chem. C 2021, 125, 7010-7021

Read Online

ABSTRACT: Material nanostructuring and optical phenomena on a nanoscale such as plasmonic effects and light scattering have been widely studied for improving the solar-to-hydrogen efficiency of photoelectrochemical (PEC) water-splitting electrodes. In this work, we report a method for analyzing the contributions of optical effects from nanostructures for enhancing the PEC performances. Electromagnetic simulations are performed for the precise calculation of generated power density in a semiconductor material. In addition, the transport and transfer of photogenerated charges to the electrolyte are modeled by using the conservation of minority carriers. The surface loss parameter, diffusion length, and doping density of the semiconductor material are determined by fitting the model to an incident photon to current efficiency (IPCE) curve

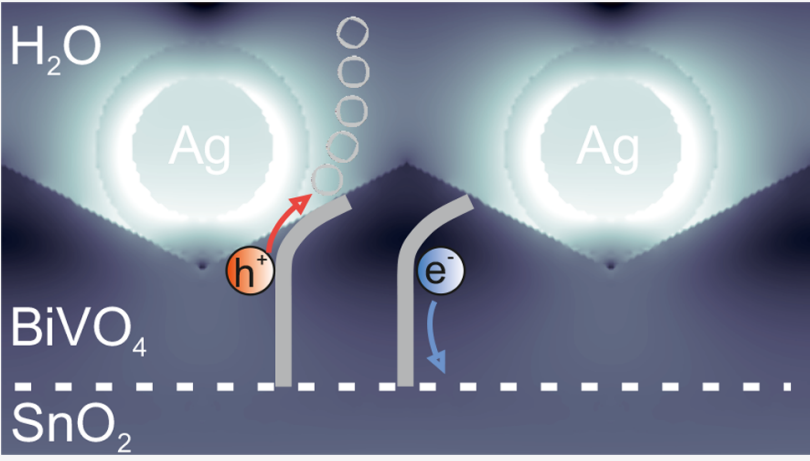
experimentally measured on the bare reference photoelectrode. These parameters are then used to compute the IPCE spectra of the photoelectrode for which an optical enhancement strategy is used, such as nanostructuring or plasmonics. The method is validated using published experimental data. The calculated IPCE enhancement ratio originating from optical effects is in quantitative agreement with experimental observations for both periodic and random optical structures. The model can be used to study in detail the key enhancement mechanisms for the IPCE from optical nanostructures and, in particular, discriminate between optical and nonoptical (e.g., catalytic) enhancement.

\section{INTRODUCTION}

Solar water splitting with photoelectrochemical (PEC) cells has the potential of being a competitive technology for hydrogen production compared to the reforming of fossil fuels. ${ }^{1}$ Intensive research is still ongoing to develop highperformance, inexpensive photoelectrodes that can operate for several years. Metal oxide semiconductors are promising candidates for practical PEC water splitting, thanks to their low cost and inherent stability under aqueous conditions. However, they face several issues that limit their solar-tohydrogen efficiency. On one hand, no metal oxide semiconductor has been found to meet both requirements of an energy band gap close to the theoretical optimum $(1.84 \mathrm{eV}$ for the top absorber in a dual stacked tandem cell $^{2}$ ) and low bulk/ surface recombinations. ${ }^{3}$ On the other hand, a thin layer thickness should be used to achieve decent charge transport because of the short minority carrier diffusion length of most metal oxides (e.g., $2-20 \mathrm{~nm}$ for $\alpha-\mathrm{Fe}_{2} \mathrm{O}_{3}{ }^{4,5}$ ) resulting in poor light absorption as a consequence of the indirect nature of the band gap. Several enhancement strategies have been elaborated. Doping of metal oxides, either during synthesis ${ }^{6}$ or with a post-treatment, ${ }^{7,8}$ has been reported as an efficient way of improving the electrical properties. Deposition of a surface catalyst such as cobalt-phosphate $(\mathrm{Co}-\mathrm{Pi})^{9}$ results in a cathodic shift of the photocurrent onset potential.
Optical effects have also been investigated to improve the performance of photoelectrodes. Thin-film phenomena have been explored, for instance, back-reflecting layers. ${ }^{10,11}$ Plasmonic nanoparticles ${ }^{12-15}$ or nanostructures ${ }^{16-18}$ combined with a water-splitting semiconductor material can yield an increased photocurrent with respect to the bare photoelectrode as a consequence of modified light absorption inside the semiconductor. Moreover, plasmonic hot carrier generation and subsequent transfer to the semiconductor has been demonstrated as a way of using light with less energy than the band gap of the semiconductor. ${ }^{19-22}$ Including light scatterers in the photoelectrode ${ }^{23,24}$ can increase the optical path of the light and be helpful for overcoming the high absorption depth of many metal oxides. Microstructured and nanostructured morphologies $^{25-29}$ and host-guest architectures ${ }^{30-35}$ where a thin semiconductor layer is deposited on a highly microstructured or nanostructured conductive guest scaffold have

Received: December 21, 2020

Revised: March 5, 2021

Published: March 26, 2021 


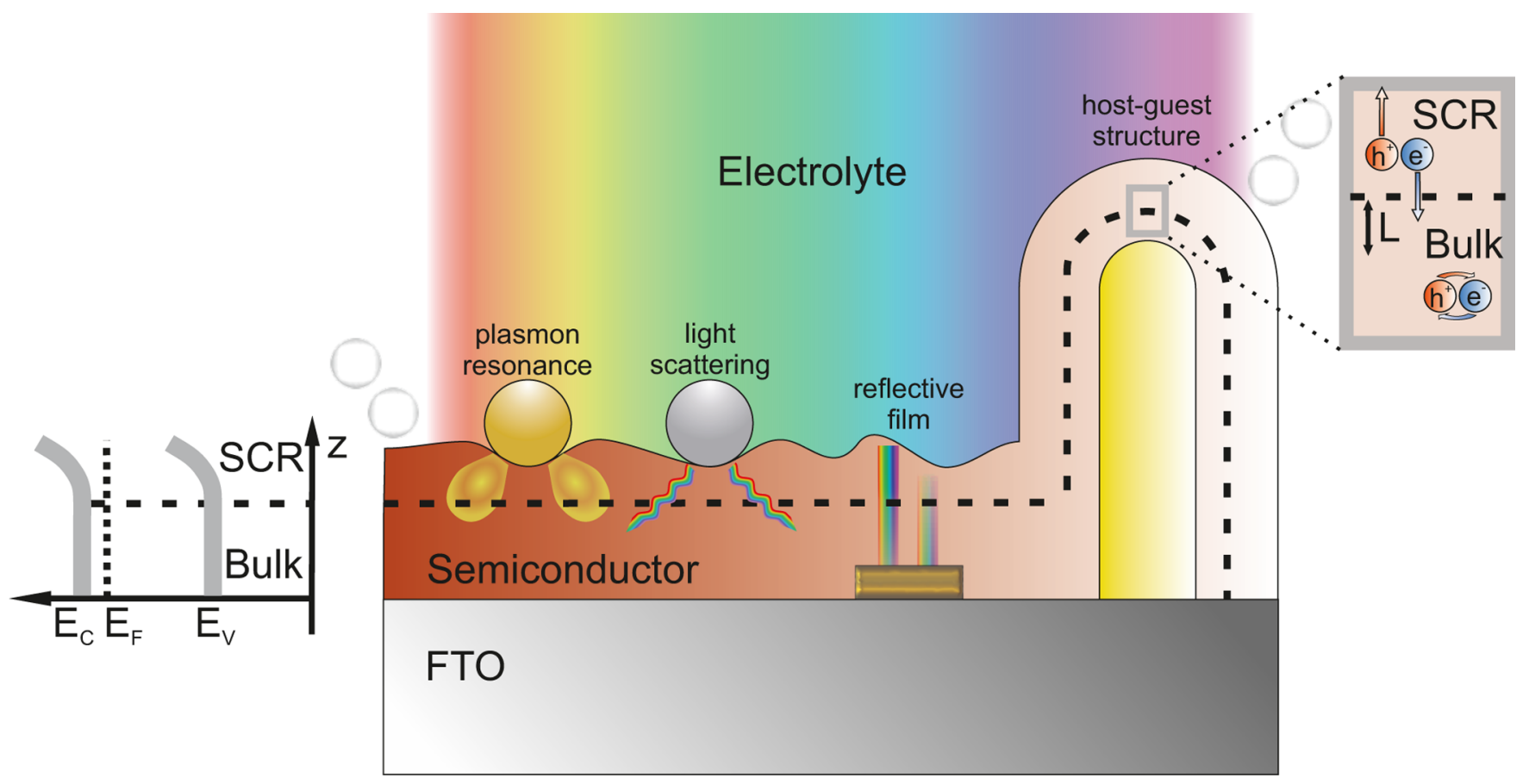

Figure 1. Sketch of an n-type semiconductor photoelectrode illuminated from the electrolyte side and different optical effects that can improve the water-splitting efficiency. The inset on the right illustrates that separation between photogenerated electrons and holes can be achieved only in the space charge region (SCR) or close to it with respect to the minority carrier diffusion length.

been widely studied. Such structures enable additional light absorption to take place close to the semiconductor/electrolyte interface due to the increased surface area with respect to a flat configuration.

Modeling approaches are important for understanding the limitations associated with the observed water-splitting performances and investigating the potential of different enhancement strategies. ${ }^{35,36}$ Gaudy and Haussener ${ }^{37}$ demonstrated a fast analysis and optimization method partially based on the work of Wilson. ${ }^{38}$ It models the incident photon to current efficiency (IPCE) and can be used to extract the optical, bulk, and surface losses of a photoelectrode from an IPCE measurement. With this tool, the authors could investigate for several materials if nanostructuring is a suitable way of improving the performances. The model assumes a flat structure of the electrode as well as a uniform light absorption in the material described by the Beer-Lambert law, which makes it unsuitable for studying heterojunctions and optical effects where the absorption profile cannot be considered as exponentially decaying (e.g., plasmonics). Dotan et al. ${ }^{10}$ investigated theoretically and experimentally optical back reflectors to enhance the photocurrent of ultrathin hematite $\left(\alpha-\mathrm{Fe}_{2} \mathrm{O}_{3}\right)$ photoanodes. A simplified model was used to account for the minority carrier's separation, transport, and transfer to the electrolyte. Moreover, by considering an ideally reflective substrate, they could obtain an analytical expression for the photon flux in the hematite layer. Encina and Coronado $^{39}$ studied plasmonic effects in isolated hybrid hematite $/$ metal $($ metal $=\mathrm{Al}, \mathrm{Ag}, \mathrm{Au})$ nanocylinders with near-field simulations. The absorption efficiency in the hematite region was simulated with discrete dipole approximation. Although the presented method provided theoretical insights into the important parameters contributing to the plasmonic enhancement, the study was purely optical and did not consider the photogenerated charge separation, transport, and transfer to the electrolyte.

In this work, we combine near-field electromagnetic simulations with a charge transport model to study optical enhancement at the nanoscale in water-splitting photoelectrodes. Precise optical modeling of the photoelectrode is performed, whereas the transport and transfer of photogenerated charges are treated semianalytically by considering the minority carriers. The presented method enables to analyze the contribution of various optical effects by considering the entire water-splitting process. In particular, optical and nonoptical contributions can be discriminated in an observed IPCE enhancement. Insights into the main mechanisms of optical enhancement are unveiled, such as its resonant or nonresonant nature, the location of electromagnetic field hot spots contributing to photocurrent generation, the influence of nanometric geometrical features on the efficiency. It is applicable to any near-field electromagnetic calculation method, thus allowing a user to choose the most appropriate and efficient approach to the system at hand.

In the following, the calculation of IPCE including optical near-field enhancement effects and a charge transport model is first described. An experimental validation of the whole method is then performed by comparing the optical enhancement calculated with the model to published data. For each case, the optical model and the optical properties of the materials are first validated by comparison with measurements. The studied geometries involve several semiconductor materials $\left(\alpha-\mathrm{Fe}_{2} \mathrm{O}_{3}\right.$ and $\left.\mathrm{BiVO}_{4}\right)$, different types of optical elements (periodically distributed host scaffold and randomly distributed nanoparticles), and different electromagnetic simulation methods (rigorous coupled wave analysis, RCWA, and surface integral equations, SIE). Finally, we discuss how the types of optical effects responsible for the observed 
enhancement can be identified from near-field studies and IPCE enhancement spectra.

\section{MODEL DESCRIPTION}

The method for calculating the IPCE (or external quantum efficiency, EQE) is described in the next section. First, the generated power density in the semiconductor material is calculated with near-field optical simulations and combined with a charge transport model to compute the EQE. In a second step, a method to analyze optical enhancement in nanostructured electrodes is presented.

Optical Simulations. Figure 1 shows different types of optical effects that have been reported to improve the performances of water-splitting photoelectrodes. ${ }^{11,15,23,31}$ Either SIE with periodic boundary conditions ${ }^{40}$ or RCWA ${ }^{41}$ is used to calculate the light intensity distribution in the semiconductor region. SIE is known to be highly robust for plasmonic near-field calculations, whereas RCWA is more adapted to large periodic dielectric structures. ${ }^{42}$ Throughout this work, the calculations are made in a unit cell containing a single feature in order to reduce computational costs. However, the presented model can be readily adapted to faster simulation techniques that enable "super cells" with a large number of features, such as the fast multipole method. ${ }^{43}$ The electric field intensity is calculated in a set of randomly generated points inside the semiconductor region. The generated power density at every point is then given by ${ }^{44}$

$$
G(\mathbf{x}, \lambda)=\frac{c}{\lambda} \pi \operatorname{Im}\left\{\varepsilon_{0} \varepsilon(\lambda)\right\}|\mathbf{E}(\mathbf{x}, \lambda)|^{2}
$$

where $c$ is the speed of light in vacuum, $\lambda$ is the vacuum wavelength, $\varepsilon_{0}$ is the vacuum permittivity, $\varepsilon=(n+\mathrm{i} k)^{2}$ is the complex permittivity of the semiconductor material, and $\mathbf{E}(\mathbf{x}, \lambda)$ is the electric field at position $\mathbf{x}$ and wavelength $\lambda$. Equation 1 will then be used in the next section to compute the EQE in the semiconductor material with and without optical nanostructuring.

IPCE Determination from Optical Simulations. The semiconductor volume can be separated into a SCR where band bending exists due to the presence of a semiconductor/ electrolyte junction (upward bending for n-type semiconductors and downward bending for p-type semiconductors) and a bulk region where no band bending exists ${ }^{45}$ (Figure 1). The study of charge separation, transport, and transfer is based on the findings of Wilson ${ }^{38}$ and Gaudy and Haussener. ${ }^{37}$ Considering that the performance is limited only by minority carriers, the EQE at a wavelength $\lambda$ can be expressed as ${ }^{37}$

$$
\operatorname{EQE}(\lambda)=\frac{R_{s}}{P_{0}}\left(\zeta \int_{V_{\mathrm{SCR}}} G(\mathbf{x}, \lambda) \mathrm{d} x+\int_{V_{\text {Bulk }}} U^{*}(\mathbf{x}) G(\mathbf{x}, \lambda) \mathrm{d} x\right)
$$

where $P_{0}$ is the incident illumination power, $R_{\mathrm{s}}=\frac{k_{\mathrm{t}}}{k_{\mathrm{t}}+k_{\mathrm{r}}}$ accounts for the surface recombination losses, with $k_{\mathrm{t}}$ and $k_{\mathrm{r}}$ being the rate constants for the charge transfer and the surface recombination, respectively. $G(\mathbf{x}, \lambda)$ is the power density of the generated electron-hole pairs at position $\mathbf{x} . V_{\mathrm{SCR}}$ and $V_{\mathrm{Bulk}}$ are the volume of the SCR and the bulk, respectively.

$U^{*}(\mathbf{x})$ is the probability that a minority carrier generated in the bulk at position $\mathbf{x}$ reaches the SCR. Its expression is derived analytically from the minority carrier conservation equation in ref 38 and is given by
$U^{*}(\mathbf{x})=\frac{L}{L+D / S} e^{-\mathrm{d}(\mathbf{x}) / L}$

with $d(\mathbf{x})$ being the distance between $\mathbf{x}$ and the edge of the SCR region, $S$ is a boundary parameter (full derivation in the Supporting Information), and $D=\mu V_{\text {th }}$ is the Einstein diffusion coefficient with $\mu$ being the mobility of the minority charge carrier.

The parameter $\zeta$ in eq 2 expresses the ratio of charges generated in the SCR that are reaching the semiconductor/ electrolyte interface ${ }^{37}$

$$
\zeta=\frac{L^{2} \phi_{\mathrm{SCR}}}{L^{2} \phi_{\mathrm{SCR}}+W_{\mathrm{SCR}}^{2} V_{\mathrm{th}}}
$$

where $L$ is the minority carrier diffusion length, $V_{\text {th }}=k_{\mathrm{B}} T / q$ is the thermal potential, and $\phi_{\mathrm{sc}}=V_{\mathrm{a}}-V_{\mathrm{fb}}$ is the potential drop across the SCR, with $V_{\mathrm{a}}$ being the applied voltage and $V_{\mathrm{fb}}$ the flat band potential of the material.

$W_{\mathrm{SCR}}$ is the thickness of the SCR defined as ${ }^{45}$

$$
W_{\mathrm{SCR}}=\sqrt{\frac{2 \varepsilon_{0} \varepsilon_{\mathrm{r}}}{q N_{\mathrm{d} / \mathrm{a}}}\left(\phi_{\mathrm{SCR}}-V_{\mathrm{th}}\right)}
$$

where $\varepsilon_{0}$ is the vacuum permittivity, $\varepsilon_{r}$ is the relative permittivity of the material, and $N_{\mathrm{d} / \mathrm{a}}$ is the density of donors/acceptors.

In the work of Gaudy and Haussener, ${ }^{37}$ the Beer-Lambert law was used to compute $G(\mathbf{x}, \lambda)$. This model was then validated on published data for several semiconductor thin films including p-type silicon, by comparing the determined diffusion length with the reported values. In this work, we study the contribution of optical nanostructures by performing a numerical calculation of $U^{*}(\mathbf{x})$ and $G(\mathbf{x}, \lambda)$ in a set of randomly generated integration points. The integrals of eq 2 are then computed numerically from the near-field distribution

$$
\begin{aligned}
\mathrm{EQE}_{\mathrm{N}}(\lambda)= & \frac{c \pi \varepsilon_{0}}{\lambda P_{0}} \operatorname{Im}\{\varepsilon(\lambda)\} R_{\mathrm{s}}\left(\zeta \frac{V_{\mathrm{SCR}}}{N_{\mathrm{SCR}}} \sum_{i=1}^{N_{\mathrm{SCR}}}\left|\mathbf{E}\left(\mathbf{x}_{\mathbf{i}}, \lambda\right)\right|^{2}\right. \\
& \left.+\frac{V_{\text {Bulk }}}{N_{\text {Bulk }}} \sum_{i=1}^{N_{\text {Bulk }}} U^{*}\left(\mathbf{x}_{\mathbf{i}}\right)\left|\mathbf{E}\left(\mathbf{x}_{\mathbf{i}}, \lambda\right)\right|^{2}\right)
\end{aligned}
$$

where $N_{\mathrm{SCR}}$ and $N_{\text {Bulk }}$ are the number of integration points in the SCR and bulk region, respectively. The values of $R_{\mathrm{s}}, L$, and $N_{\mathrm{d} / \mathrm{a}}$ in this equation are chosen according to direct or indirect characterization. The size parameters of the photoelectrode and the optical constants of the materials are determined from a prior characterization of the electrode structure and a comparison of the simulated optical properties (transmission, absorption, absorbance, etc.) with measurements. The EQE of a photoelectrode including optical nanostructures can then be calculated and compared to the bare photoelectrode. This will be discussed in detail in the section "Optical Enhancement Calculation".

Our charge transport model considers that only the electron/hole pairs generated in the SCR or close to the SCR with respect to the minority carrier diffusion length contribute to the calculated EQE (Figure 1). This aspect is a key factor to optimize optical contributions to the photocatalytic performances and is not considered when only the overall light absorption in the semiconductor region is studied, as in ref 39. 


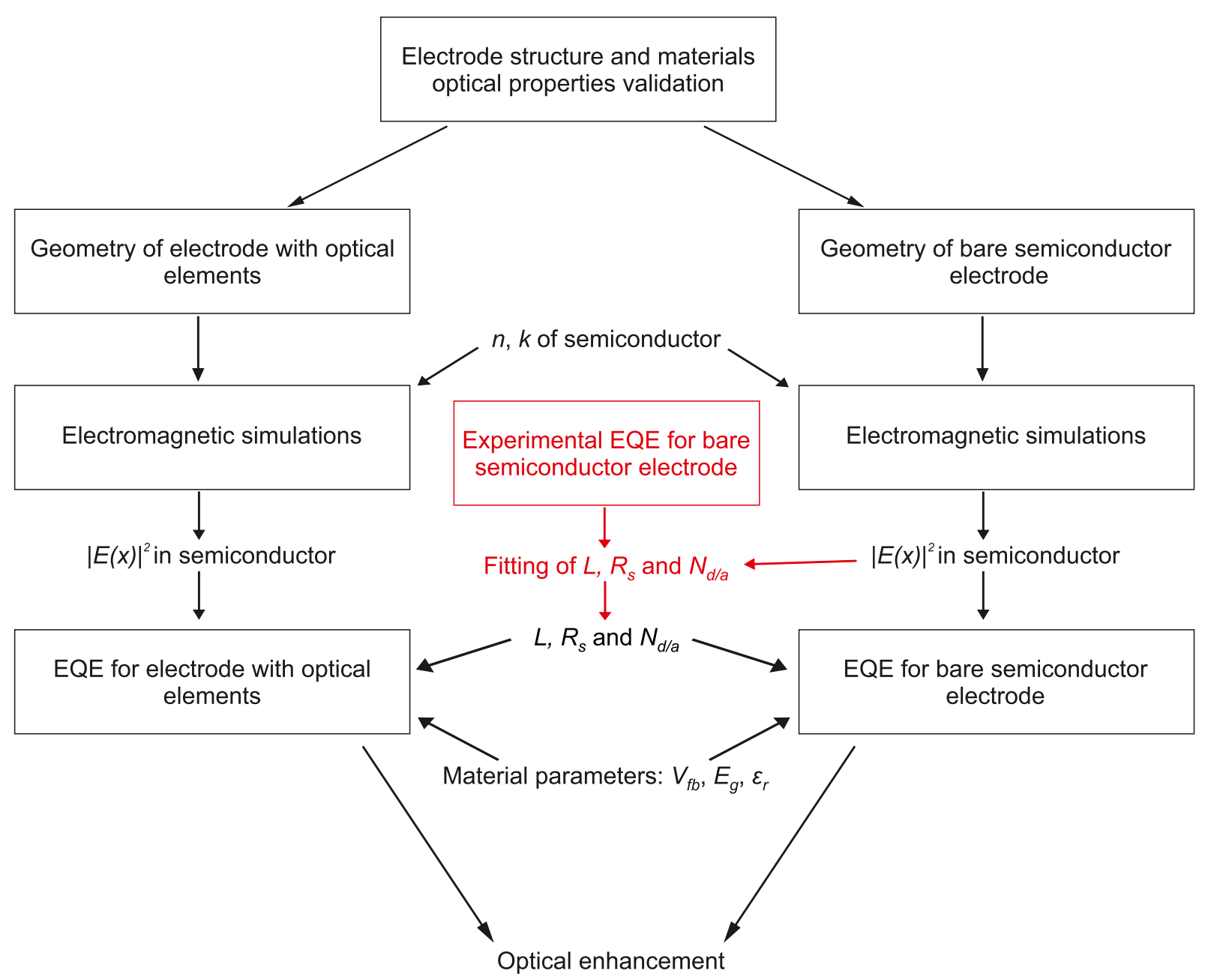

Figure 2. Flowchart of the procedure for computing the optical enhancement between a hybrid photoelectrode including optical elements and a bare photoelectrode. The steps in red determine $R_{\mathrm{S}}, L$, and $N_{\mathrm{d} / \mathrm{a}}$ from an experimental EQE curve measured on the bare photoelectrode. These steps do not need to be performed if these parameters are already known for the considered electrode.

Scope. Majority carrier transport and transfer are not taken into account; therefore, the description given by the model is not accurate if these parameters are the limiting performance factors. Minority carrier recombinations at the back contact of the photoelectrode (e.g., due to defect state at the FTO/ semiconductor interface) are also not considered. Experimentally, they can be drastically reduced by depositing a blocking layer for minority carriers between the substrate and the semiconductor (such as $\mathrm{WO}_{3}{ }^{46}$ or $\mathrm{SnO}_{2}{ }^{47}$ ). Back contact recombinations are also negligible when the electrode is illuminated from the electrolyte side (front illumination), if the minority carrier diffusion length is smaller than the semiconductor thickness.

It is assumed that the addition of optical elements modifies only the light absorption profile in the semiconductor and has no effect on the charge separation, charge transport, and transfer efficiency. Optical enhancement is defined as the improvement of PEC performances (EQE, photocurrent) due to optical effects. Any photocurrent or/and EQE enhancement described with our model is an optical enhancement. Therefore, the overall predicted enhancement can be different from the experimentally observed one. Reduced surface losses can be expected when the added optical elements have a surface catalytic effect (e.g., when they are made of precious metal $^{11,15}$ ) or are able to locally heat the semiconductor or the electrolyte, such as when plasmonic nanoparticles are used. ${ }^{14,48}$ In these cases, the EQE given by the model can be compared with experimental measurements where the charge transfer efficiency can be assumed to be $100 \%$ and reduced surface losses due to the optical elements do not play a role. Depositing an additional catalyst on the surface of the photoelectrode (e.g., Co-Pi) or using an electrolyte that contains a sacrificial substance (hole/electron scavenger for photoanodes/photocathodes) can help achieving such a configuration. In a hole/electron scavenger, the assumption of $100 \%$ charge transfer efficiency is only valid at high applied potentials for metal oxide electrodes. ${ }^{49,50}$ Fitting the charge transfer parameter $R_{\mathrm{s}}$ (section "Optical Enhancement Calculation") can indicate if the assumption is valid or not. The technique of combining EQE measurements in electrolytes with and without hole scavengers has already been reported for experimentally discriminating the catalytic and optical contributions coming from the addition of metallic nanoparticles. $^{13,24,51}$ However, modified charge separation and transport in the hybrid structure cannot be differentiated from purely optical enhancement.

We assumed in eq 6 that $100 \%$ of the absorbed photons generate useful charge carriers for water oxidation/reduction. However, it has been recently reported for some materials that not all optical transitions contributing to the absorption 
spectrum generate mobile charge carriers. ${ }^{52-54}$ To account for this phenomenon, eq 6 can be multiplied with the photogeneration yield spectrum if it is known for the material considered. The minority carrier mobility $\mu$ (expression of the Einstein diffusion coefficient in eq 3 ) in the considered semiconductor is not required to be known if the applied voltage $V_{\mathrm{a}}$ is such that the SCR potential is larger than 0.23 $\mathrm{V}^{37}$ This is the case for all examples studied in the next section; therefore, a constant value of $\mu$ was used. The SCR potential is assumed to be unperturbed by the incident light, and a uniform donors/acceptors concentration is assumed in the semiconductor. Finally, the model does not account for the plasmonic hot electron injection mechanism ${ }^{15,19,21}$ and can be therefore combined with the theoretical frameworks developed for hot electron generation ${ }^{55}$ for analyzing plasmonic EQE enhancement and discriminating between hot electron injection and near-field effects.

Optical Modeling of Materials and Geometry. The optical model must be compared to optical measurements before considering the optoelectronic properties of the system. This validation can be performed in various ways, depending on the optical enhancement strategy chosen. In this work, we will investigate two type of strategies: host-guest nanostructuring of the substrate and addition of nanoparticles. The parameters of the simulated geometry and the optical constants of the materials considered should be chosen to realistically describe the fabricated sample. This needs to be investigated through a careful characterization of the fabricated structure and comparison of the simulated optical properties (transmission, absorption, absorbance...) with experimental measurements. The simulated geometry should include the interfaces that are optically not negligible (e.g., semiconductor/substrate). The porosity of the semiconductor layer can be considered optically using an effective medium approximation for the permittivity of the semiconductor. ${ }^{56}$

In the section " $\mathrm{Ti} / \mathrm{Fe}_{2} \mathrm{O}_{3}$ Film on the Nanostructured Substrate", the designed geometry is validated by comparing the model with the measured absorption spectra

$$
A(\lambda)=1-R(\lambda)-T(\lambda)
$$

where $R(\lambda)$ and $T(\lambda)$ are the reflectance and transmittance spectra, respectively.

In the section " $\mathrm{BiVO}_{4}$ Electrode with $\mathrm{Ag} @ \mathrm{SiO}_{2}$ Nanoparticles", the nanoparticles are not considered perfectly monodispersed and we use a linear combination of several nanoparticle sizes in the EQE calculations. The measured distribution is used to select the sizes, and we impose that the most occurring value has the highest weight in the distribution. The coefficients are determined by fitting the measured absorbance with a linear combination of extinction efficiency spectra, defined as

$$
Q_{\text {ext }}(\lambda)=\frac{C_{\mathrm{abs}}(\lambda)+C_{\mathrm{sca}}(\lambda)}{\pi R^{2}}
$$

where $C_{\mathrm{abs}}(\lambda)$ and $C_{\mathrm{sca}}(\lambda)$ are the absorption and scattering cross sections, respectively, and $\pi R^{2}$ is the physical cross section of the nanoparticle.

Optical Enhancement Calculation. A semi-analytical method for the calculation of EQE has been established. We report now on a procedure to compute the optical enhancement by nanostructures (Figure 2). Optical simulations for the bare photoelectrode (reference sample with no specific optical enhancement strategy) and the hybrid photoelectrode (for which an optical enhancement strategy is used) are performed first, from which the power density of the photogenerated electron/hole pairs in the semiconductor layer is calculated (eq $1)$. As input for calculation of EQE, the parameters $V_{\mathrm{fb}}, E_{\mathrm{g}}$, and $\varepsilon_{r}$ should be provided (Table S1) as well as the surface recombination parameter should be provided as well as the surface recombination parameter $R_{S}$, the minority carrier diffusion length $L$, and the donors/acceptors doping density $N_{\mathrm{d} / \mathrm{a}}$. These three last parameters can be determined by fitting eq 6 to an experimental EQE curve measured on the bare photoelectrode such that the optimal values maximize the coefficient $R^{2}$

$$
R^{2}=1-\frac{\sum_{i}\left|\mathrm{EQE}_{\exp }\left(\lambda_{i}\right)-\mathrm{EQE}_{\mathrm{N}}\left(\lambda_{i} ; R_{\mathrm{S}}, L, N_{\mathrm{d} / \mathrm{a}}\right)\right|^{2}}{\sum_{i}\left|\mathrm{EQE}_{\text {exp }}\left(\lambda_{i}\right)-\overline{\mathrm{EQE}}_{\text {exp }}\right|^{2}}
$$

where $\operatorname{EQE}_{\exp }\left(\lambda_{i}\right)$ is the experimentally measured EQE at wavelength $\lambda_{i}, \mathrm{EQE}_{\mathrm{N}}$ is given by eq 6 and $\overline{\mathrm{EQE}_{\exp }}$ is the average value of the experimental EQE from the lowest measurement wavelength $\lambda_{0}$ to the wavelength corresponding to the band gap of the semiconductor. If one or several of these parameters are already known, this procedure can be performed with the unknown parameters only. The values of $R_{S}, L$, and $N_{\mathrm{d} / \mathrm{a}}$ are assumed to be identical for the bare and hybrid photoelectrodes. For all simulations, the value of hole mobility $\mu$ and charge transfer rate constant $k_{\mathrm{t}}$ were assumed as in ref 37 to be $1 \mathrm{~cm}^{2} \mathrm{~V}^{-1} \mathrm{~s}^{-1}$ and $10^{-2} \mathrm{~cm} \mathrm{~s}^{-1}$, respectively. The EQE spectra for bare and hybrid photoelectrodes are calculated with eq 6 from the input parameters and the values of $|E(\mathbf{x})|$ in the semiconductor obtained with electromagnetic simulations. The photocurrent enhancement between the hybrid and bare sample is calculated from the EQE spectra

$$
\frac{J_{\mathrm{h}}}{J_{\mathrm{b}}}=\frac{\int \mathrm{EQE}_{2}(\lambda) \cdot \operatorname{AM} 1.5 \mathrm{G}(\lambda) \mathrm{d} \lambda}{\int \mathrm{EQE}_{1}(\lambda) \cdot \operatorname{AM} 1.5 \mathrm{G}(\lambda) \mathrm{d} \lambda}
$$

where $\operatorname{AM1.5G}(\lambda)$ is the solar spectrum, $\operatorname{EQE}_{2}(\lambda)$ is the $\mathrm{EQE}$ spectrum of the hybrid sample, and $\mathrm{EQE}_{1}(\lambda)$ is the $\mathrm{EQE}$ spectrum of the bare sample. Photocurrent enhancement being a result of integration of the entire spectrum, a proper model validation can be first sought through comparison with EQE measurement.

Finally, the geometry, material, and relative position of the optical elements and semiconductor layer in the hybrid system can be optimized through several iterations.

\section{RESULTS AND DISCUSSION}

In the following, the model is applied to experimentally fabricated photoelectrodes previously reported where optical effects from nanostructures were claimed to be the origin of the observed EQE enhancement. The investigated systems involve two different metal oxide materials (hematite and bismuth vanadate), different strategies for optical enhancement (periodic nanostructuring and randomly distributed metallic nanoparticles), and different optical simulation methods (SIE and RCWA) in order to demonstrate the broad applicability of the method. Simulated data are compared to reported measurements to confirm the validity of the model. We illustrate through these examples that this model allows us to clearly and nonambiguously identify the origin of optical enhancement. In particular, the contribution of plasmon resonances is compared to nonresonant scattering. 
a
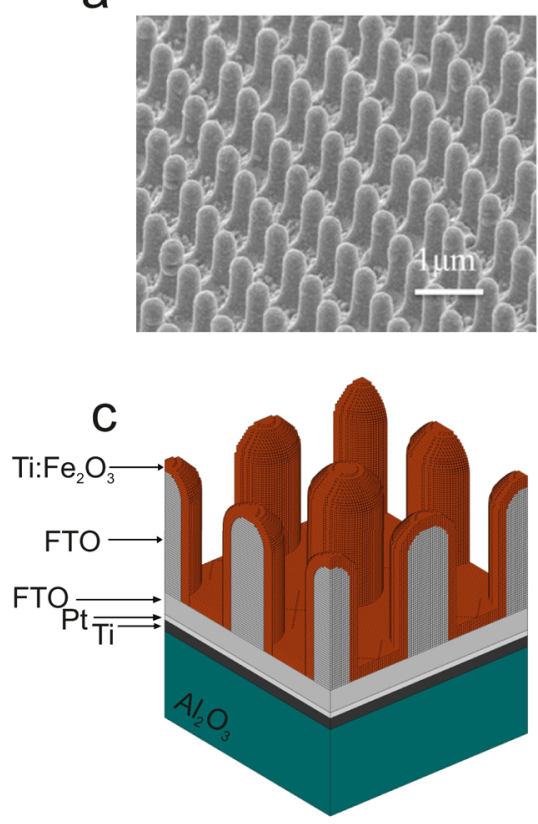

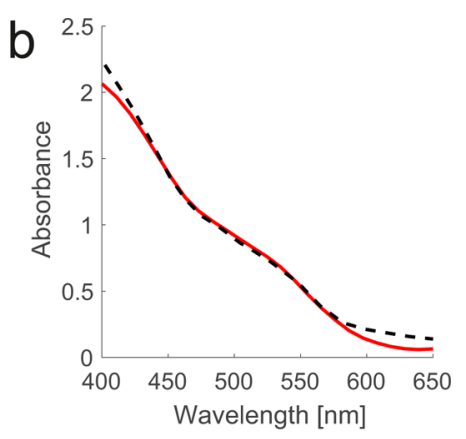

d

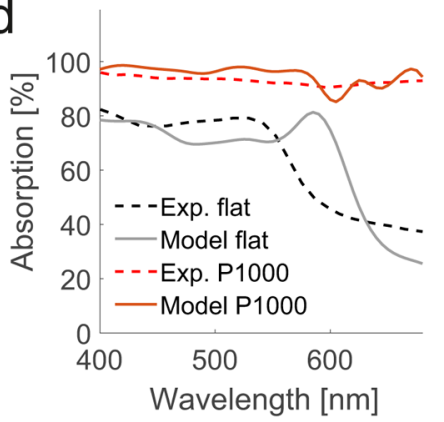

Figure 3. (a) SEM image of a fabricated sample where $\mathrm{Ti} / \mathrm{Fe}_{2} \mathrm{O}_{3}$ was deposited on a nanostructured FTO scaffold. Reprinted (adapted) with permission from ref 57. Copyright 2014 American Chemical Society. (b) Calculated absorbance of a $100 \mathrm{~nm}$ hematite layer deposited on FTOcoated glass (plain curve), compared with measurements from ref 57 (dashed curve). (c) Scheme of the periodic design used for simulations of the nanostructured sample with a $100 \mathrm{~nm}$ hematite film. (d) Absorption spectra calculated for the flat and nanostructured cases, compared with measurements from ref 57 .

$\mathrm{Ti} / \mathrm{Fe}_{2} \mathrm{O}_{3}$ Film on the Nanostructured Substrate. We consider first a host-guest structure where a thin film of Tidoped hematite $\left(\mathrm{Ti} / \mathrm{Fe}_{2} \mathrm{O}_{3}\right)$ is deposited on a nanostructured FTO scaffold. Qiu et al. ${ }^{57}$ demonstrated an increased EQE and photocurrent for such a nanostructure compared to the planar case. The scaffold was made of nanopillars arranged in a periodic square pattern, and the electrode was illuminated from the electrolyte side (front illumination). The EQE and photocurrent were measured by Qiu et al. ${ }^{57}$ in $1 \mathrm{M} \mathrm{NaOH}$ aqueous electrolyte without a sacrificial hole scavenger. Therefore, our analysis will help to discriminate between optical and nonoptical enhancement. We performed the optical simulations of these structures with RCWA. The size parameters used to design the simulated geometry were extracted from the SEM images shown in ref 57 (device with $1000 \mathrm{~nm}$ period shown in Figure 3a). The thickness of the Ti/ $\mathrm{Fe}_{2} \mathrm{O}_{3}$ was chosen to be $100 \mathrm{~nm}$ by calculating the absorbance of a layer deposited on FTO-coated glass and comparing with the measurements from ref 57 (Figure $3 \mathrm{~b}$ ). The optical constants of hematite were taken from ref 53, and the optical constants of FTO and glass were taken from ref 58. The simulated geometry is shown in Figure 3c. The optical constants of $\mathrm{Al}_{2} \mathrm{O}_{3}, \mathrm{Ti}$, and $\mathrm{Pt}$ were taken from refs, ${ }^{59,60,53}$ respectively. Structures having periods of 1000 and $1500 \mathrm{~nm}$ were investigated. In agreement with the SEM images shown by Qiu et al., ${ }^{57}$ the height of the FTO pillars was set to $110 \%$ of the period and their diameter was set to $40 \%$ of the period. The calculated absorption spectra of the flat and nanostructured geometry with $1000 \mathrm{~nm}$ period (Figure 3d) agree reasonably well with the measurements from ref 57 . In particular, the optical model shows correctly that the nanostructured sample has almost $100 \%$ absorption in the whole visible range. The peak at $580 \mathrm{~nm}$ in the simulated spectrum for the flat configuration originates from a thin-film resonance in the FTO layer. Its spectral position varies with the angle of incidence (Figure S1a) and it disappears when FTO is replaced by platinum (Figure S1b). The roughness of the deposited films, which is not considered in the modeled geometry, could explain that it is not observed in the measured spectrum.

In the next step, the parameters $N_{\mathrm{d}} L$, and $R_{\mathrm{s}}$ of the $\mathrm{Ti} /$ $\mathrm{Fe}_{2} \mathrm{O}_{3}$ film were deduced by fitting the simulated EQE for the planar configuration to the experimentally measured curve in the $1 \mathrm{M} \mathrm{NaOH}$ aqueous electrolyte. It was recently shown that not all optical transitions in hematite are able to generate useful charge carriers for water oxidation. ${ }^{52,53}$ To account for that, eq 6 was multiplied by the wavelength-dependent photogeneration yield of hematite experimentally measured by Segev et al. ${ }^{53}$ (Figure S2). The fitting procedure for the planar (bare) configuration gave $R^{2}=0.97$ (eq 9). The value of $L, N_{\mathrm{d}}$, and $R_{\mathrm{s}}$ are $4.1 \mathrm{~nm}, 2.16 \times 10^{19} \mathrm{~cm}^{-3}$, and 0.75 , respectively. The diffusion length found is within the reported data for $\alpha-\mathrm{Fe}_{2} \mathrm{O}_{3}$. ${ }^{4,5}$ However, the donor density $N_{\mathrm{d}}$ is higher than the published values for pure hematite, ${ }^{61,62}$ and this can be explained by the high Ti-doping $\left(\mathrm{Ti} / \mathrm{Fe}\right.$ ratio of $3: 35^{57}$ ). Samples with a large $\mathrm{Ti}$ fraction were reported to have a higher donor concentration than the undoped material. ${ }^{62}$

The simulated EQE for the nanostructured (hybrid) sample with $1000 \mathrm{~nm}$ period (Figure 4a) is in overall good spectral and quantitative agreement with the measured data from ref 57. The model predicts the EQE to be slightly higher than the experimental one in the range $360-430 \mathrm{~nm}$ and a bit lower in the interval $450-530 \mathrm{~nm}$. This mismatch could originate from the deviation in the absorption spectrum for the flat configuration (Figure 3d) and also from the difference between the photogeneration yield spectra of pure hematite and $\mathrm{Ti} /$ $\mathrm{Fe}_{2} \mathrm{O}_{3}$. The EQE spectra in ref 57 were measured from $360 \mathrm{~nm}$ 

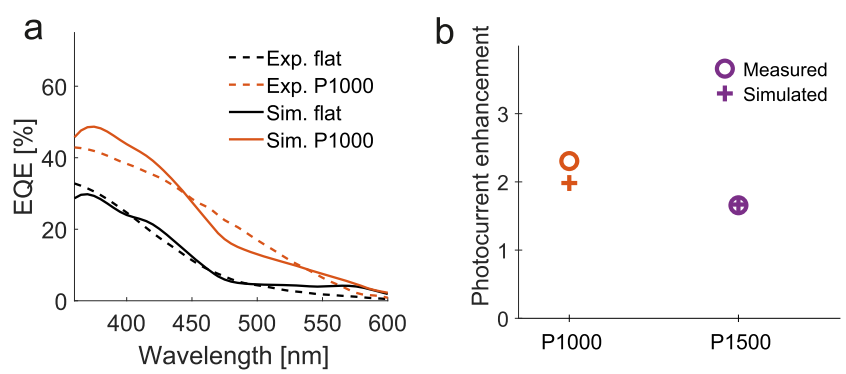

Figure 4. (a) Simulated EQE of the structure with $100 \mathrm{~nm}$ hematite deposited on planar and nanostructured substrates, compared with the experimental results obtained by Qiu et al. ${ }^{57}$ The simulated curve for the planar configuration represents the best fit to the measured data. The simulated EQE for the structure with $1500 \mathrm{~nm}$ period is shown in Figure S3. (b) Photocurrent enhancement between the nanostructured and flat samples (calculated using eq 10) compared to the experimental values extracted from photocurrent measurements in ref 57.

whereas the absorption and absorbance spectra (Figure $3 \mathrm{~b}, \mathrm{~d}$ ) are shown only from $400 \mathrm{~nm}$.

Figure $4 \mathrm{~b}$ shows the photocurrent enhancement between the nanostructured (hybrid) and planar (bare) samples. A very good agreement with enhancement obtained from photocurrent measurement was obtained for the sample with 1000 $\mathrm{nm}$ period (2.3/2.0 for measurement/simulations), whereas a perfect agreement was obtained for the sample with $1500 \mathrm{~nm}$ period. This suggests that the nanostructured scaffold does not have an effect in improving the charge transfer efficiency, hole diffusion length, or SCR size but contributes only through optical effects. As a conclusion, our method showed correctly that the PEC performances of the structure with $1000 \mathrm{~nm}$ period is higher than the one with $1500 \mathrm{~nm}$ period and provided a quantitative agreement with EQE and photocurrent measurements from ref 57.
$\mathrm{BiVO}_{4}$ Electrode with $\mathrm{Ag} @ \mathrm{SiO}_{2}$ Nanoparticles. In the previous section, periodic nanostructuring has been studied as an example. Now, in order to show the broad applicability of the method, we consider plasmonic nanoparticles. Abdi et al. ${ }^{24}$ have reported that the EQE of a $\sim 100 \mathrm{~nm}$ thick $\mathrm{BiVO}_{4}$ photoelectrode could be increased by adding $\mathrm{Ag} @ \mathrm{SiO}_{2}$ nanoparticles at the surface of the semiconductor, in the configuration where the electrode is illuminated from the substrate side (back illumination). The $\mathrm{EQE}$ enhancement ratio between the samples with and without $\mathrm{Ag} @ \mathrm{SiO}_{2}$ nanoparticles was found to be much higher in a pure buffer than in an electrolyte containing an additional hole scavenger, which suggests that the contribution of the $\mathrm{Ag} @ \mathrm{SiO}_{2}$ nanoparticles is not only optical. Measurements with an additional hole scavenger electrolyte were therefore used for comparison with our model which studies optical enhancement. The optical properties of these structures were calculated with SIE simulations. A unit cell of the simulated geometry and sketches of the fabricated samples are shown in Figure $5 \mathrm{a}-\mathrm{c}$. The $\mathrm{SnO}_{2}$ underlayer serves as a hole blocking layer ${ }^{47}$ and prevents hole recombinations at the back contact. Electron transport is known as a limiting factor of performances in $\mathrm{BiVO}_{4}$, but this was specifically identified for thicker $\mathrm{BiVO}_{4}$ films than in this example (evidenced from $200 \mathrm{~nm}$ in ref 46). In addition, the electrode is illuminated from the back side, which results in most electrons being generated close to the back contact. This justifies the applicability of our model which does not consider majority carrier transport and recombinations at the back-contact. The chosen period and roughness (modeled in a periodic way) were chosen to match realistically the AFM data shown in ref 24 .

The exact $\mathrm{BiVO}_{4}$ film thickness and the dimensions of the nanoparticles were chosen by comparing our optical model to the measured data by Abdi et al. ${ }^{24}$ The absorbance of the bare structure (without nanoparticles) was first calculated (Figure

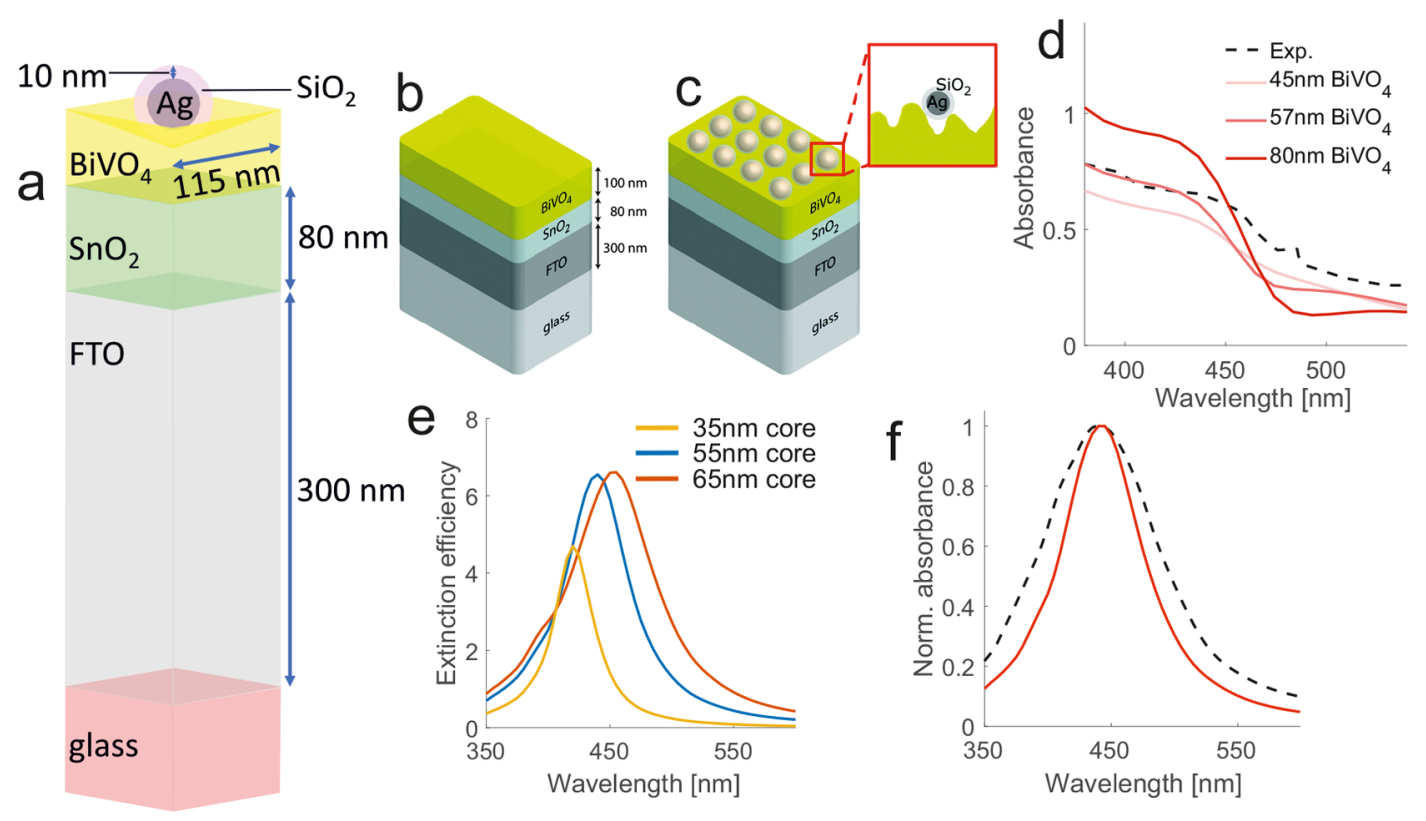

Figure 5. (a) Scheme of the simulated unit cell for the hybrid sample. (b,c) Scheme of the bare $\mathrm{BiVO}_{4}$ sample (b) and the hybrid sample containing $\mathrm{Ag} @ \mathrm{SiO}_{2}$ nanoparticles (c), reproduced with permission from ref 24. Copyright 2014 Royal Society of Chemistry. (d) Calculated absorbance of the bare sample for different $\mathrm{BiVO}_{4}$ film thicknesses and comparison with measurements from ref 24 (e) Extinction efficiency spectra of $\mathrm{Ag} @ \mathrm{SiO}_{2}$ nanoparticles in water with a shell thickness of $10 \mathrm{~nm}$. (f) Comparison of absorbance calculated as a linear combination of the three spectra of Figure 5e (plain red curve) with the measured absorbance from ref 24 (dashed curve). 
5d). The optical constants of $\mathrm{BiVO}_{4}$ were taken from ref 63, and the optical constants of $\mathrm{SnO}_{2}, \mathrm{FTO}$, and glass were taken from ref 58. The measured absorbance was well reproduced when a $57 \mathrm{~nm} \mathrm{BiVO}_{4}$ film was simulated, especially in the range $380-450 \mathrm{~nm}$ where $\mathrm{BiVO}_{4}$ has a high absorption. This value of the thickness is realistic with respect to the AFM measurements shown in ref 24. The size of the silver nanoparticles was determined by assuming a constant shell thickness of $10 \mathrm{~nm}$ and using the method described in the section "Optical Modeling of Materials and Geometry". The size distribution of the nanoparticles reported in ref 24 was used, with the constraint that the most occurring size $(55 \mathrm{~nm})$ has the highest weight in the linear combination. The optical constants of silver were taken from ref 64 . It was found that the combination of 35,55 , and $65 \mathrm{~nm}$ core sizes (Figure 5e) reproduces reasonably the measured absorbance from ref 24 (Figure 5f). The narrower peak could be due to the nonperfect spherical shape of the nanoparticles in reality. A comparison with the size distribution measured by Abdi et al. ${ }^{24}$ is shown in Figure S4.

The parameters $N_{\mathrm{d}}, L$, and $R_{\mathrm{s}}$ were determined by fitting the calculated $\mathrm{EQE}$ for the bare $\mathrm{BiVO}_{4}$ electrode to the experimental curve in the hole scavenger electrolyte. The fitting procedure was performed for the three film thicknesses that were tested in Figure 5d, and the optimized parameters are shown in Table 1 . It can be seen that for all three values a

Table 1. Determined Material Parameter for Different Film Thicknesses

\begin{tabular}{lclll} 
& $\mathrm{L}[\mathrm{nm}]$ & $N_{\mathrm{D}}\left[\mathrm{cm}^{-3}\right]$ & \multicolumn{1}{c}{$R_{\mathrm{S}}$} & $R^{2}$ \\
$45 \mathrm{~nm} \mathrm{BiVO}_{4}$ & 33.2 & $2.5 \times 10^{18}$ & 0.98 & 0.96 \\
$57 \mathrm{~nm} \mathrm{BiVO}_{4}$ & 13.5 & $2.7 \times 10^{18}$ & 1 & 0.95 \\
$80 \mathrm{~nm} \mathrm{BiVO}_{4}$ & 13.1 & $1.4 \times 10^{18}$ & 0.98 & 0.91 \\
\hline
\end{tabular}

reasonable fitting quality can be achieved $\left(R^{2}\right.$ values from 0.91 to 0.96). However, the value of the optimized parameters differs. As the $57 \mathrm{~nm} \mathrm{BiVO}$ film best reproduces the measured absorbance, the optimized value of $N_{\mathrm{d}}, L$, and $R_{\mathrm{s}}$ can be trusted only in this case to match the properties of the fabricated $\mathrm{BiVO}_{4}$, showing the importance of a prior validation of the photoelectrode optical properties.

The fitted EQE for the bare sample, simulated EQE for the hybrid sample and photocurrent enhancement are shown in Figure 6 and compared with PEC measurements from the work of Abdi et al. ${ }^{24}$ The photocurrent enhancement was calculated with eq 10. The fitting procedure for the bare sample was performed only in the interval 400-540 nm. Indeed quality of the fit was poorer when the whole range 350-540 nm was considered (Figure S5). It should be mentioned that experimental errors are higher in the UV spectral range, where solar light and artificial light sources used for EQE measurements irradiate less than in the visible region. Therefore, performing the fitting procedure in the interval 400-540 nm has a small impact on the calculated photocurrent. The entire range $350-540 \mathrm{~nm}$ was considered for calculating the photocurrent enhancement, but the EQE spectra used is the best fit in the interval $400-540 \mathrm{~nm}$. A weighted average between the curves obtained with 35,55 , and $65 \mathrm{~nm}$ core nanoparticles was performed to calculate the EQE of the hybrid samples, using the coefficients found in Figure $5 \mathrm{f}$ (given in Figure S4). The average filling factor is $31 \%$, which matches realistically with the SEM images of the fabricated samples shown in the work of Abdi et al. ${ }^{24}$ The EQE curves for the single nanoparticle sizes are shown in Figure S6.

First, it can be seen qualitatively that the positive contribution of $\mathrm{Ag} @ \mathrm{SiO}_{2}$ nanoparticles to the PEC performances is well reproduced by the model. The structure including a $57 \mathrm{~nm}$ film thickness gives an overall lower EQE than experimentally, except in the range 470-550 nm which contributes less to the photocurrent. However, the calculated photocurrent enhancement is similar to the experimental value calculated from photocurrent measurements (1.26/1.15 for measurements/simulations). There is an important difference between the photocurrent enhancements derived from measured EQE spectra (1.39) and photocurrent (1.26), which indicates that the difference between model and measurements is in the range of measurement uncertainties. When the $\mathrm{BiVO}_{4}$ film thickness is decreased to $50 \mathrm{~nm}$, the simulated EQE for the hybrid sample matches relatively well the experimental observations. Finally, both the simulated EQE and the photocurrent enhancement are higher than the experimental data when a $45 \mathrm{~nm}$ film is considered. In general, these results show that the predicted enhancement can be higher, similar, or lower than the experimental observations when a small variation in the $\mathrm{BiVO}_{4}$ thickness is considered (12 $\mathrm{nm}$ difference between the thinnest and the thickest configuration). This could possibly be due to a slightly different $\mathrm{BiVO}_{4}$ thickness between the hybrid and bare samples or to some thickness variation across samples coming from the
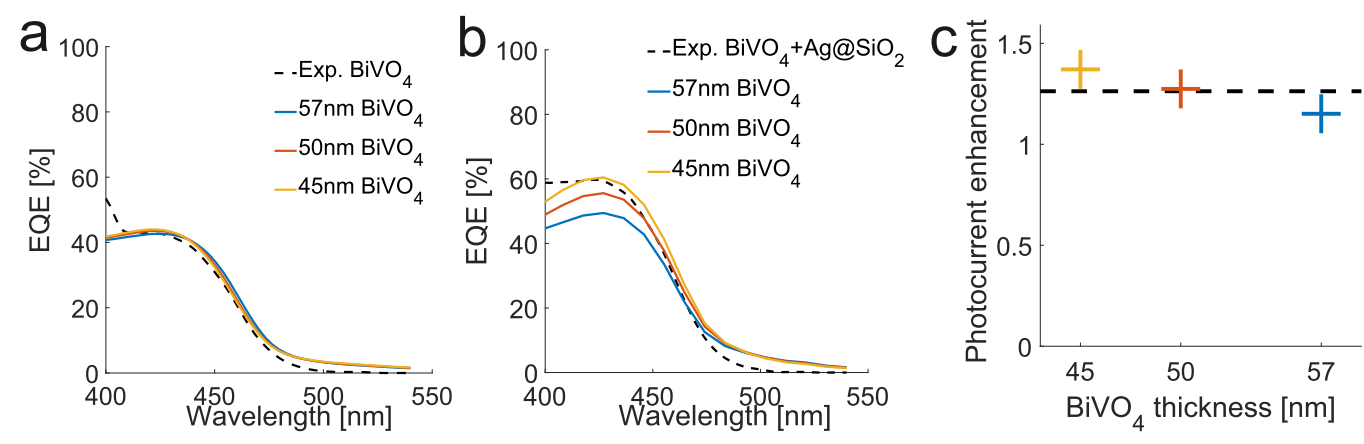

Figure 6. (a) Best EQE fit for the bare sample, for different $\mathrm{BiVO}_{4}$ film thicknesses. (b) Simulated EQEs for the hybrid sample, compared to the measured data in the hole scavenger electrolyte from Abdi et al. ${ }^{44}$ They were calculated as a weighted average of the curves corresponding to nanoparticles with 35, 55, and $65 \mathrm{~nm}$ core, using the same coefficients as in Figure 5f. (c) Photocurrent enhancement between the hybrid and bare samples (calculated using eq 10) for different $\mathrm{BiVO}_{4}$ film thicknesses and comparison with the experimental value calculated from the measured photocurrent in the hole scavenger electrolyte (dashed curve) in ref 24. 
deposition method (spray pyrolysis ${ }^{65}$ ). As a conclusion, the results given by our model agree quantitatively with both $\mathrm{EQE}$ and photocurrent measurements within experimental uncertainties. This is a remarkable result given that we use periodic boundary conditions to describe a system of randomly distributed nanoparticles.

Figure 7a shows the EQE enhancement spectrum for $50 \mathrm{~nm}$ $\mathrm{BiVO}_{4}$ thickness resulting from simulations and comparison
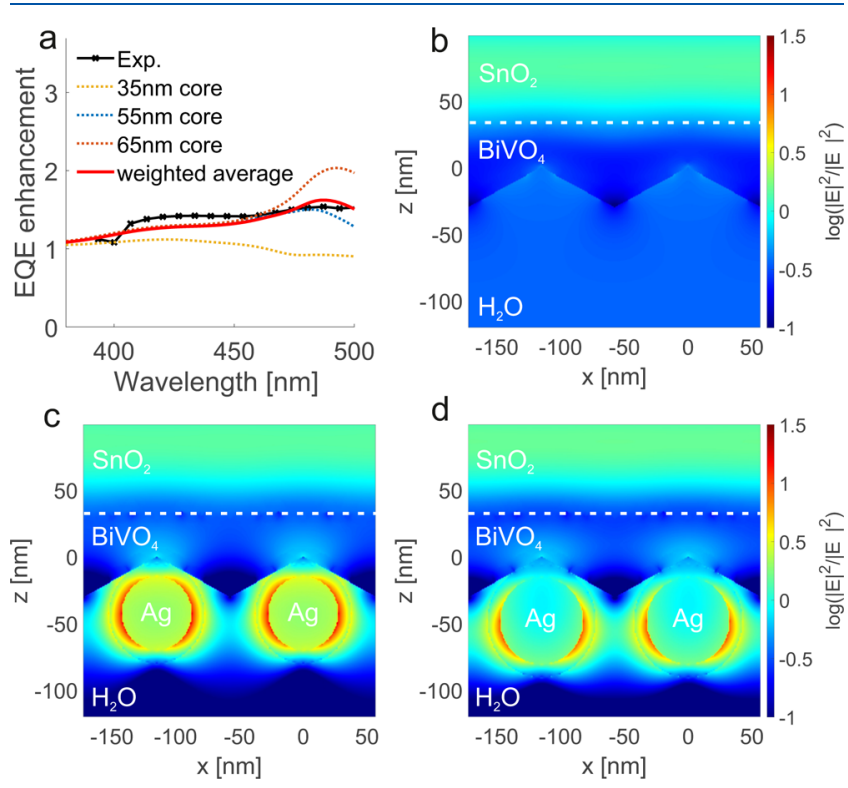

Figure 7. (a) EQE enhancement spectra of the hybrid and bare $\mathrm{BiVO}_{4}$ samples with $50 \mathrm{~nm}$ film thickness corresponding to single nanoparticle sizes and their weighted average. $(b-d)$ Electric field intensity map at $427 \mathrm{~nm}$ for the (b) bare sample, (c) hybrid sample with $55 \mathrm{~nm}$ silver core nanoparticles, and (d) hybrid sample with 65 $\mathrm{nm}$ silver core nanoparticles. The electric field intensity map for the $35 \mathrm{~nm}$ core nanoparticles is shown in Figure S7. The light is normally incident and polarized on the plane of the cross section.

with the experimental observation. A good general spectral agreement with the measurements is observed, showing that the lower EQE for the hybrid sample at short wavelength with respect to the experimental curve (Figure 6b) is a consequence of the same phenomenon happening for the bare sample below $415 \mathrm{~nm}$ (Figure 6a) which has been discussed previously. The spectra corresponding to single nanoparticle sizes show resonant features at 427, 470, and $490 \mathrm{~nm}$ for the 35,55 , and $65 \mathrm{~nm}$ silver core, respectively. This coincides with the plasmon resonance of $\mathrm{Ag} @ \mathrm{SiO}_{2}$ nanoparticles placed at the interface between water and $\mathrm{BiVO}_{4}$.

We now analyze the origin of the optical enhancement. The plasmon resonance of 55 and $65 \mathrm{~nm}$ core nanoparticles is at a longer wavelength compared to the absorption maximum of BiVO4, whereas the peak for $35 \mathrm{~nm}$ nanoparticles matches. Interestingly, 55 and $65 \mathrm{~nm}$ nanoparticles yield a higher enhancement in the high absorption region of BiVO4, which let us think that both resonant (plasmonic) and nonresonant scattering contribute to the enhancement. Indeed, the field intensity in the $\mathrm{BiVO}_{4}$ layer is lower in Figure $7 \mathrm{~b}$ than in $7 \mathrm{c}, \mathrm{d}$, as a result of the interaction between light and nanoparticles off-resonance.

In order to study the contribution of the resonant scattering originating from plasmonic properties with respect to nonresonant scattering, structures including gold and silver nanoparticles with $65 \mathrm{~nm}$ core and $10 \mathrm{~nm} \mathrm{SiO}$ shell were compared (Figure 8a). The EQE obtained with $\mathrm{Au} @ \mathrm{SiO}_{2}$ nanoparticles is much lower compared to $\mathrm{Ag} @ \mathrm{SiO}_{2}$ and almost coincides with the EQE of the bare structure. Figure $8 \mathrm{~b}$ shows that the plasmon resonance of $\mathrm{Au} @ \mathrm{SiO}_{2}$ nanoparticles is at a longer wavelength compared to the absorption maximum of $\mathrm{BiVO}_{4}$ but no significant EQE enhancement is observed in the

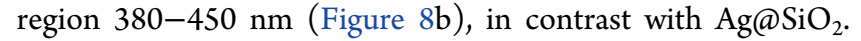
As visible in Figure 8c, there is not much interaction between light and nanoparticles, resulting in a field intensity in $\mathrm{BiVO}_{4}$ comparable with the bare structure (Figure $7 \mathrm{~b}$ ). These results enable to conclude that the plasmonic properties of silver highly contribute to the enhancement mechanism.

\section{CONCLUSIONS}

We presented a method for studying optical enhancement from nanostructures in water-splitting photoelectrodes and its contribution to the photocatalytic performances. It involves precise optical modeling, computation of the light distribution in the photoelectrode, and a simplified treatment of charge carrier separation, transport, and transfer. This enables accurate description of the optical effects while limiting the computational cost. In combination with experimental measurements in an electrolyte containing sacrificial hole/ electron scavengers, the model was demonstrated to be a powerful tool for discriminating between optical and nonoptical enhancements. The method was validated on several experimentally fabricated photoelectrodes. A very good qualitative and quantitative agreement between the calculated and measured optical enhancement of the PEC performances was obtained for both a periodic and a random structure. We
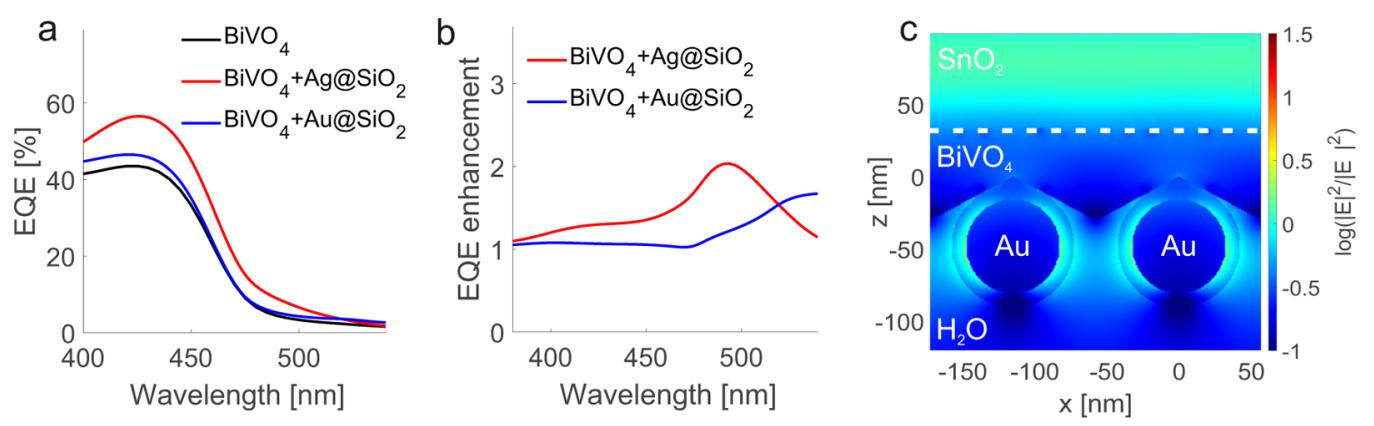

Figure 8. (a) Simulated $\mathrm{EQE}$ for a bare $\mathrm{BiVO}_{4}$ sample with $50 \mathrm{~nm}$ film thickness and hybrid samples including either $\mathrm{Ag} @ \mathrm{SiO}_{2}$ or $\mathrm{Au} @ \mathrm{SiO} \mathrm{O}_{2}$ with $65 \mathrm{~nm}$ core and $10 \mathrm{~nm}$ shell. (b) EQE enhancement spectra between the hybrid and bare samples. (c) Electric field intensity map for the $\mathrm{BiVO}_{4}+$ $\mathrm{Au} @ \mathrm{SiO}_{2}$ sample at $427 \mathrm{~nm}$. The light is normally incident and polarized on the plane of the cross section. 
also showed the impact of structural parameters on the enhancement. It is applicable to a wide variety of systems and optical simulation methods. In particular, we showed the contribution of resonant plasmonic and nonresonant scattering to the optical enhancement. Our analysis has finally revealed that thorough material and optical characterization is required in order to achieve agreement with experiments. In the future, the development of fast simulation methods that enables precise near-field computations will increase the possibilities for optimizing complicated geometries and materials combinations. This work will provide the opportunity for researchers to elaborate efficient strategies for optically improving the solar-to-hydrogen efficiency of water-splitting devices and to understand the origin of the optical effects involved when improved performances are observed.

\section{ASSOCIATED CONTENT}

\section{(s) Supporting Information}

The Supporting Information is available free of charge at https://pubs.acs.org/doi/10.1021/acs.jpcc.0c11342.

Derivation of the boundary parameter $S$; input material parameters for hematite and bismuth vanadate; thin-film resonance for the flat hematite photoelectrode; photogeneration yield spectrum used in the study of the hematite photoanode; EQE of the $\mathrm{Ti} / \mathrm{Fe}_{2} \mathrm{O}_{3}$ nanostructure with $1500 \mathrm{~nm}$ period; experimental and modeled $\mathrm{Ag} @ \mathrm{SiO}_{2}$ nanoparticle size distribution; EQE of the bismuth vanadate sample fitted between 350 and 530 $\mathrm{nm}$; EQEs for the hybrid samples $\mathrm{BiVO}_{4}+\mathrm{Ag} @ \mathrm{SiO}_{2}$ corresponding to single nanoparticle dimensions; and field intensity map for the $35 \mathrm{~nm}$ silver core nanoparticle (PDF)

\section{AUTHOR INFORMATION}

\section{Corresponding Authors}

Benjamin Gallinet - CSEM Muttenz, Muttenz CH-4132,

Switzerland; 이이.org/0000-0001-7444-3398;

Email: bgt@csem.ch

Edwin C. Constable - Department of Chemistry, University of Basel, Basel CH-4058, Switzerland;

Email: edwin.constable@unibas.ch

\section{Authors}

Luc Driencourt - Department of Chemistry, University of Basel, Basel CH-4058, Switzerland; CSEM Muttenz, Muttenz CH-4132, Switzerland; Swiss Nanoscience Institute, Basel 4056, Switzerland; 이이.org/0000-0001-75210465

Catherine E. Housecroft - Department of Chemistry, University of Basel, Basel CH-4058, Switzerland; () orcid.org/0000-0002-8074-0089

Sören Fricke - CSEM Muttenz, Muttenz CH-4132, Switzerland

Complete contact information is available at:

https://pubs.acs.org/10.1021/acs.jpcc.0c11342

\section{Notes}

The authors declare no competing financial interest.

\section{ACKNOWLEDGMENTS}

We would like to acknowledge funding from the Swiss Nanoscience Institute. Also, we would particularly like to thank the referees of an earlier version of this paper for their valuable suggestions.

\section{REFERENCES}

(1) Nowotny, J.; Bak, T.; Chu, D.; Fiechter, S.; Murch, G. E.; Veziroglu, T. N. Sustainable practices: Solar hydrogen fuel and education program on sustainable energy systems. Int. J. Hydrogen Energy 2014, 39, 4151-4157.

(2) Pinaud, B. A.; Benck, J. D.; Seitz, L. C.; Forman, A. J.; Chen, Z.; Deutsch, T. G.; James, B. D.; Baum, K. N.; Baum, G. N.; Ardo, S.; et al. Technical and economic feasibility of centralized facilities for solar hydrogen production via photocatalysis and photoelectrochemistry. Energy Environ. Sci. 2013, 6, 1983-2002.

(3) Kim, J. H.; Hansora, D.; Sharma, P.; Jang, J.-W.; Lee, J. S. Toward practical solar hydrogen production - an artificial photosynthetic leaf-to-farm challenge. Chem. Soc. Rev. 2019, 48, 19081971.

(4) Kennedy, J. H.; Frese, K. W. Photooxidation of Water at $\alpha$ - Fe2 O 3 Electrodes. J. Electrochem. Soc. 1978, 125, 709-714.

(5) Dare-Edwards, M. P.; Goodenough, J. B.; Hamnett, A.; Trevellick, P. R. Electrochemistry and photoelectrochemistry of iron(III) oxide. J. Chem. Soc., Faraday Trans. 1 1983, 79, 2027-2041.

(6) Parmar, K. P. S.; Kang, H. J.; Bist, A.; Dua, P.; Jang, J. S.; Lee, J. S. Photocatalytic and Photoelectrochemical Water Oxidation over Metal-Doped Monoclinic BiVO4Photoanodes. ChemSusChem 2012, 5, 1926-1934.

(7) Hao, Y.; Deng, J.; Zhou, L.; Sun, X.; Zhong, J. Depth-reduction induced low onset potential of hematite photoanodes for solar water oxidation. RSC Adv. 2015, 5, 31086-31090.

(8) Kim, J. H.; Lee, J. S. Elaborately Modified BiVO4Photoanodes for Solar Water Splitting. Adv. Mater. 2019, 31, 1806938.

(9) Zhong, D. K.; Cornuz, M.; Sivula, K.; Grätzel, M.; Gamelin, D. R. Photo-assisted electrodeposition of cobalt-phosphate (Co-Pi) catalyst on hematite photoanodes for solar water oxidation. Energy Environ. Sci. 2011, 4, 1759-1764.

(10) Dotan, H.; Kfir, O.; Sharlin, E.; Blank, O.; Gross, M.; Dumchin, I.; Ankonina, G.; Rothschild, A. Resonant light trapping in ultrathin films for water splitting. Nat. Mater. 2012, 12, 158-164.

(11) Zhao, J.; Guo, Y.; Cai, L.; Li, H.; Wang, K. X.; Cho, I. S.; Lee, C. H.; Fan, S.; Zheng, X. High-performance ultrathin BiVO4 photoanode on textured polydimethylsiloxane substrates for solar water splitting. ACS Energy Lett. 2016, 1, 68-75.

(12) Archana, P. S.; Pachauri, N.; Shan, Z.; Pan, S.; Gupta, A. Plasmonic enhancement of photoactivity by gold nanoparticles embedded in hematite films. J. Phys. Chem. C 2015, 119, 1550615516.

(13) Valenti, M.; Dolat, D.; Biskos, G.; Schmidt-Ott, A.; Smith, W. A. Enhancement of the photoelectrochemical performance of CuWO4 thin films for solar water splitting by plasmonic nanoparticle functionalization. J. Phys. Chem. C 2015, 119, 2096-2104.

(14) Valenti, M.; Kontoleta, E.; Digdaya, I. A.; Jonsson, M. P.; Biskos, G.; Schmidt-Ott, A.; Smith, W. A. The Role of Size and Dimerization of Decorating Plasmonic Silver Nanoparticles on the Photoelectrochemical Solar Water Splitting Performance of BiVO4Photoanodes. ChemNanoMat 2016, 2, 739-747.

(15) Mascaretti, L.; Dutta, A.; Kment, Š.; Shalaev, V. M.; Boltasseva, A.; Zbořil, R.; Naldoni, A. Plasmon-Enhanced Photoelectrochemical Water Splitting for Efficient Renewable Energy Storage. Adv. Mater. 2019, 31, 1805513.

(16) Gao, H.; Liu, C.; Jeong, H. E.; Yang, P. Plasmon-enhanced photocatalytic activity of iron oxide on gold nanopillars. ACS Nano 2012, 6, 234-240.

(17) Li, J.; Cushing, S. K.; Zheng, P.; Meng, F.; Chu, D.; Wu, N. Plasmon-induced photonic and energy-transfer enhancement of solar water splitting by a hematite nanorod array. Nat. Commun. 2013, 4, 2651.

(18) Kim, J. K.; Shi, X.; Jeong, M. J.; Park, J.; Han, H. S.; Kim, S. H.; Guo, Y.; Heinz, T. F.; Fan, S.; Lee, C.-L.; et al. Enhancing Mo:BiVO4 
solar water splitting with patterned $\mathrm{Au}$ nanospheres by plasmoninduced energy transfer. Adv. Energy Mater. 2018, 8, 1701765.

(19) Knight, M. W.; Sobhani, H.; Nordlander, P.; Halas, N. J. Photodetection with active optical antennas. Science 2011, 332, 702704.

(20) Mukherjee, S.; Libisch, F.; Large, N.; Neumann, O.; Brown, L. V.; Cheng, J.; Lassiter, J. B.; Carter, E. A.; Nordlander, P.; Halas, N. J. Hot Electrons Do the Impossible: Plasmon-Induced Dissociation of H2on Au. Nano Lett. 2013, 13, 240-247.

(21) Mubeen, S.; Lee, J.; Singh, N.; Krämer, S.; Stucky, G. D.; Moskovits, M. An autonomous photosynthetic device in which all charge carriers derive from surface plasmons. Nat. Nanotechnol. 2013, $8,247-251$.

(22) Chen, Y.-C.; Hsu, Y.-K.; Popescu, R.; Gerthsen, D.; Lin, Y.-G.; Feldmann, C. Au@Nb@HxK1-xNbO3 nanopeapods with nearinfrared active plasmonic hot-electron injection for water splitting. Nat. Commun. 2018, 9, 232.

(23) Zhang, L.; Herrmann, L. O.; Baumberg, J. J. Size dependent plasmonic effect on BiVO4 photoanodes for solar water splitting. Sci. Rep. 2015, 5, 16660.

(24) Abdi, F. F.; Dabirian, A.; Dam, B.; Van De Krol, R. Plasmonic enhancement of the optical absorption and catalytic efficiency of BiVO4 photoanodes decorated with $\mathrm{Ag} @ \mathrm{SiO} 2$ core-shell nanoparticles. Phys. Chem. Chem. Phys. 2014, 16, 15272-15277.

(25) Warren, S. C.; Voïtchovsky, K.; Dotan, H.; Leroy, C. M.; Cornuz, M.; Stellacci, F.; Hébert, C.; Rothschild, A.; Grätzel, M. Identifying champion nanostructures for solar water-splitting. Nat. Mater. 2013, 12, 842-849.

(26) Kim, J. Y.; Magesh, G.; Youn, D. H.; Jang, J.-W.; Kubota, J.; Domen, K.; Lee, J. S. Single-crystalline, wormlike hematite photoanodes for efficient solar water splitting. Sci. Rep. 2013, 3, 2681.

(27) Chen, H. M.; Chen, C. K.; Liu, R.-S.; Zhang, L.; Zhang, J.; Wilkinson, D. P. Nano-architecture and material designs for water splitting photoelectrodes. Chem. Soc. Rev. 2012, 41, 5654-5671.

(28) Bora, D. K.; Braun, A.; Erni, R.; Fortunato, G.; Graule, T.; Constable, E. C. Hydrothermal treatment of a hematite film leads to highly oriented faceted nanostructures with enhanced photocurrents. Chem. Mater. 2011, 23, 2051-2061.

(29) Sivula, K.; Le Formal, F.; Grätzel, M. Solar Water Splitting: Progress Using Hematite $(\alpha$-Fe2O3) Photoelectrodes. ChemSusChem 2011, 4, 432-449.

(30) Sivula, K.; Formal, F. L.; Grätzel, M.S. WO3-Fe2O3Photoanodes for Water Splitting: A Host Scaffold, Guest Absorber Approach. Chem. Mater. 2009, 21, 2862-2867.

(31) Rao, P. M.; Cai, L.; Liu, C.; Cho, I. S.; Lee, C. H.; Weisse, J. M.; Yang, P.; Zheng, X. Simultaneously Efficient Light Absorption and Charge Separation in WO3/BiVO4Core/Shell Nanowire Photoanode for Photoelectrochemical Water Oxidation. Nano Lett. 2014, 14, 1099-1105.

(32) Pihosh, Y.; Turkevych, I.; Mawatari, K.; Uemura, J.; Kazoe, Y.; Kosar, S.; Makita, K.; Sugaya, T.; Matsui, T.; Fujita, D.; et al. Photocatalytic generation of hydrogen by core-shell WO3/BiVO4 nanorods with ultimate water splitting efficiency. Sci. Rep. 2015, 5, 11141 .

(33) Boudoire, F.; Toth, R.; Heier, J.; Braun, A.; Constable, E. C. Photonic light trapping in self-organized all-oxide microspheroids impacts photoelectrochemical water splitting. Energy Environ. Sci. 2014, 7, 2680-2688.

(34) Qiu, Y.; Liu, W.; Chen, W.; Chen, W.; Zhou, G.; Hsu, P.-C.; Zhang, R.; Liang, Z.; Fan, S.; Zhang, Y.; et al. Efficient solar-driven water splitting by nanocone BiVO4-perovskite tandem cells. Sci. Adv. 2016, 2, No. e1501764.

(35) Suter, S.; Graf, R.; Moreno García, D.; Haussener, S. Optimizing and implementing light trapping in thin-film, mesostructured photoanodes. ACS Appl. Mater. Interfaces 2020, 12, 5739-5749.

(36) Gaudy, Y. K.; Haussener, S. Utilizing modeling, experiments, and statistics for the analysis of water-splitting photoelectrodes. J. Mater. Chem. A 2016, 4, 3100-3114.
(37) Gaudy, Y. K.; Haussener, S. Rapid performance optimization method for photoelectrodes. J. Phys. Chem. C 2019, 123, 2183821851.

(38) Wilson, R. H. A model for the current-voltage curve of photoexcited semiconductor electrodes. J. Appl. Phys. 1977, 48, 4292-4297.

(39) Encina, E. R.; Coronado, E. A. Keys for designing hematite/ plasmonic metal hybrid nanostructures with enhanced photoactive properties. J. Phys. Chem. C 2018, 122, 4589-4599.

(40) Gallinet, B.; Kern, A. M.; Martin, O. J. F. Accurate and versatile modeling of electromagnetic scattering on periodic nanostructures with a surface integral approach. J. Opt. Soc. Am. A 2010, 27, 22612271.

(41) Moharam, M. G.; Gaylord, T. K.; Grann, E. B.; Pommet, D. A. Formulation for stable and efficient implementation of the rigorous coupled-wave analysis of binary gratings. J. Opt. Soc. Am. A 1995, 12, $1068-1076$.

(42) Gallinet, B.; Butet, J.; Martin, O. J. F. Numerical methods for nanophotonics: standard problems and future challenges. Laser Photonics Rev. 2015, 9, 577-603.

(43) Solís, D. M.; Taboada, J. M.; Obelleiro, F.; Liz-Marzán, L. M.; García de Abajo, F. J. Toward ultimate nanoplasmonics modeling. ACS Nano 2014, 8, 7559-7570.

(44) Kern, A. M.; Martin, O. J. F. Pitfalls in the determination of optical cross sections from surface integral equation simulations. IEEE Trans. Antennas Propag. 2010, 58, 2158-2161.

(45) Van de Krol, R.; Grätzel, M. Photoelectrochemical Hydrogen Production; Springer US, 2012.

(46) Chen, L.; Alarcón-Lladó, E.; Hettick, M.; Sharp, I. D.; Lin, Y.; Javey, A.; Ager, J. W. Reactive sputtering of bismuth vanadate photoanodes for solar water splitting. J. Phys. Chem. C 2013, 117, 21635-21642.

(47) Liang, Y.; Tsubota, T.; Mooij, L. P. A.; Van De Krol, R. Highly improved quantum efficiencies for thin film BiVO4 photoanodes. J. Phys. Chem. C 2011, 115, 17594-17598.

(48) Zhang, L.; Ye, X.; Boloor, M.; Poletayev, A.; Melosh, N. A.; Chueh, W. C. Significantly enhanced photocurrent for water oxidation in monolithic Mo:BiVO4/SnO2/Si by thermally increasing the minority carrier diffusion length. Energy Environ. Sci. 2016, 9, 2044-2052.

(49) Dotan, H.; Sivula, K.; Grätzel, M.; Rothschild, A.; Warren, S. C. Probing the photoelectrochemical properties of hematite $(\alpha-\mathrm{Fe} 2 \mathrm{O} 3)$ electrodes using hydrogen peroxide as a hole scavenger. Energy Environ. Sci. 2011, 4, 958-964.

(50) Zhong, D. K.; Choi, S.; Gamelin, D. R. Near-complete suppression of surface recombination in solar photoelectrolysis by "Co-Pi" catalyst-modified W:BiVO4. J. Am. Chem. Soc. 2011, 133, 18370-18377.

(51) Jiang, C.-M.; Segev, G.; Hess, L. H.; Liu, G.; Zaborski, G.; Toma, F. M.; Cooper, J. K.; Sharp, I. D. Composition-dependent functionality of copper vanadate photoanodes. ACS Appl. Mater. Interfaces 2018, 10, 10627-10633.

(52) Hayes, D.; Hadt, R. G.; Emery, J. D.; Cordones, A. A.; Martinson, A. B. F.; Shelby, M. L.; Fransted, K. A.; Dahlberg, P. D.; Hong, J.; Zhang, X.; et al. Electronic and nuclear contributions to time-resolved optical and X-ray absorption spectra of hematite and insights into photoelectrochemical performance. Energy Environ. Sci. 2016, 9, 3754-3769.

(53) Segev, G.; Dotan, H.; Ellis, D. S.; Piekner, Y.; Klotz, D.; Beeman, J. W.; Cooper, J. K.; Grave, D. A.; Sharp, I. D.; Rothschild, A. The spatial collection efficiency of charge carriers in photovoltaic and photoelectrochemical cells. Joule 2018, 2, 210-224.

(54) Wiktor, J.; Reshetnyak, I.; Strach, M.; Scarongella, M.; Buonsanti, R.; Pasquarello, A. Sizable excitonic effects undermining the photocatalytic efficiency of $\beta$-Cu2V2O7. J. Phys. Chem. Lett. 2018, 9, 5698-5703.

(55) Manjavacas, A.; Liu, J. G.; Kulkarni, V.; Nordlander, P. Plasmon-induced hot carriers in metallic nanoparticles. ACS Nano 2014, 8, 7630-7638. 
(56) Khardani, M.; Bouaïcha, M.; Bessaïs, B. Bruggeman effective medium approach for modelling optical properties of porous silicon: comparison with experiment. Phys. Status Solidi C 2007, 4, 19861990.

(57) Qiu, Y.; Leung, S.-F.; Zhang, Q.; Hua, B.; Lin, Q.; Wei, Z.; Tsui, K.-H.; Zhang, Y.; Yang, S.; Fan, Z. Efficient photoelectrochemical water splitting with ultrathin films of hematite on three-dimensional nanophotonic structures. Nano Lett. 2014, 14, 2123-2129.

(58) Cendula, P.; Steier, L.; Losio, P. A.; Grätzel, M.; Schumacher, J. $\mathrm{O}$. Analysis of optical losses in a photoelectrochemical cell: A tool for precise absorptance estimation. Adv. Funct. Mater. 2018, 28, 1702768.

(59) Boidin, R.; Halenkovič, T.; Nazabal, V.; Beneš, L.; Němec, P. Pulsed laser deposited alumina thin films. Ceram. Int. 2016, 42, $1177-1182$.

(60) Johnson, P.; Christy, R. Optical constants of transition metals: Ti, V, Cr, Mn, Fe, Co, Ni, and Pd. Phys. Rev. B: Solid State 1974, 9, 5056-5070.

(61) Tamirat, A. G.; Rick, J.; Dubale, A. A.; Su, W.-N.; Hwang, B.-J. Using hematite for photoelectrochemical water splitting: a review of current progress and challenges. Nanoscale Horiz. 2016, 1, 243-267.

(62) Yan, D.; Tao, J.; Kisslinger, K.; Cen, J.; Wu, Q.; Orlov, A.; Liu, $\mathrm{M}$. The role of the domain size and titanium dopant in nanocrystalline hematite thin films for water photolysis. Nanoscale 2015, 7, 1851518523.

(63) Cooper, J. K.; Gul, S.; Toma, F. M.; Chen, L.; Liu, Y.-S.; Guo, J.; Ager, J. W.; Yano, J.; Sharp, I. D. Indirect bandgap and optical properties of monoclinic bismuth vanadate. J. Phys. Chem. C 2015, 119, 2969-2974.

(64) Palik, E. D. Handbook of Optical Constants of Solids; Elsevier, 1985.

(65) Abdi, F. F.; Firet, N.; van de Krol, R. Efficient BiVO4 thin film photoanodes modified with cobalt phosphate catalyst and W-doping. ChemCatChem 2013, 5, 490-496. 